\title{
Phase-dependent Spontaneous Spin Polarization and Bifurcation Delay in Coupled Two-Component Bose-Einstein Condensates
}

\author{
Chaohong Lee \\ Max Planck Institute for the Physics of Complex Systems, Noethnitzer Str. 38, D-01187 Dresden, \\ Germany, and \\ Wuhan Institute of Physics and Mathematics, The Chinese Academy of Sciences, Wuhan 430071, \\ P. R. China \\ Wenhua Hai \\ Department of Physics, Hunan Normal University, Changsha 410081,P. R. China \\ Lei Shi, and Kelin Gao \\ Wuhan Institute of Physics and Mathematics, The Chinese Academy of Sciences, Wuhan 430071, \\ P. R. China
}

(November 4, 2018)

\begin{abstract}
The spontaneous spin polarization and bifurcation delay in two-component Bose-Einstein condensates coupled with laser or/and radio-frequency pulses are investigated. We find that the bifurcation and the spontaneous spin polarization are determined by both physical parameters and relative phase between two condensates. Through bifurcations, the system enters into the spontaneous spin polarization regime from the Rabi regime. We also find that bifurcation delay appears when the parameter is swept through a static bifur-
\end{abstract}

*Corresponding author: chlee@mpipks-dresden.mpg.de and chleecn@hotmail.com. 
cation point. This bifurcation delay is responsible for metastability leading to hysteresis.

PACS numbers: 03.75.Fi, 42.65.Pc, 02.30.Oz, 75.60.Nt 


\section{INTRODUCTION}

Electronic and nuclear-spin polarization in an atomic vapor with optical pumping have been investigated extensively [1]. Under conditions in which electronic spin exchange takes place faster than spin relaxation, spontaneous spin polarization appears. This interesting phenomenon is very similar to ferromagnetism and has been observed in wide ranges of atomic intensity, pump laser frequency and intensity. The appearance of spontaneous spin polarization means that the atomic vapor has two stable states with large spin polarization. The experimental realization of it has been applied to the field of optical bistability [2]. The atomic spin polarization exhibits striking hysteresis in switching between the bistable states [1]. This is analogs to those ferromagnetic system displays magnetic hysteresis [3].

With more and more deeply exploring the mechanism of the spontaneous spin polarization phenomena in thermal atomic gases, it raises a question of whether the spontaneous spin polarization in ultracold atomic gases is as same as the one in thermal gases. Recently, the experimental realization of multi-component Bose-Einstein condensates (BECs) $[4,5]$ in different hyperfine levels causes our interest in considering the similar behavior of the ultracold atomic gases. There are many distinguishable differences between thermal atomic gases and cold ones. The first one is that the collision among thermal atomic gases is noncoherent. However, when the temperature is close to the critical temperature realizing Bose-Einstein condensate $(\mathrm{BEC})\left(T \sim T_{B E C}\right)$ or below it, the collision among ultracold atomic gases is coherent due to the path between such collision is smaller than the phase coherence length $[7,8]$. Can this coherent property play a important role in the polarization process of the ultracold atoms? Another distinct difference is that the interaction strength of cold atoms can be controlled easily $[7,9]$. But the interaction strength in the thermal case is very difficult to control.

In this article, we shall show the coherence among ultracold atoms gives rise to the phasedependent spontaneous spin polarization and bifurcation which depend on both the physical parameters and the relative phase. In the next section, using the variational approach, we 
gain the model from the mean-field description of laser coupled Bose-Einstein condensates in different hyperfine levels of the same kind of atoms. Then, based upon the obtained model, we analyze the parameter-dependence and the phase-dependence of the spontaneous spin polarization from the points of bifurcation. We simultaneously find that bifurcation delay, which is relative to bistability/metastability, appears when related parameters slowly sweep through static bifurcation points. Lastly, we take a brief look at experimental possibilities. In the third section, we summarize and discuss the obtained results.

\section{PHASE-DEPENDENT SPONTANEOUS SPIN POLARIZATION AND BIFURCATION DELAY}

We consider that the same kind of bosonic atoms, which are trapped in a single-well potential, are condensed in two different hyperfine levels $\mid 1>$ and $\mid 2>$. Raman transitions or two-photon transitions between two hyperfine states are induced by the laser fields with the effective Rabi-frequency $\Omega$ and a finite detuning $\delta$. The internal Josephson effects $[4,5,10-13]$, coherent coupling effects [14], vortices [15] and spin waves [16] in such systems have stimulated great interest of many theoretists and experimentalists. In the rotating frame, neglecting damping and finite-temperature effects, this coupled two-component BECs system can be described by a pair of coupled GPEs $[11,14,15]$

$$
\begin{aligned}
& i \hbar \frac{\partial \Psi_{2}(\vec{r}, t)}{\partial t}=\left(H_{2}^{0}+H_{2}^{M F}-\frac{\delta}{2}\right) \Psi_{2}(\vec{r}, t)+\frac{\Omega}{2} \Psi_{1}(\vec{r}, t), \\
& i \hbar \frac{\partial \Psi_{1}(\vec{r}, t)}{\partial t}=\left(H_{1}^{0}+H_{1}^{M F}+\frac{\delta}{2}\right) \Psi_{1}(\vec{r}, t)+\frac{\Omega}{2} \Psi_{2}(\vec{r}, t) .
\end{aligned}
$$

Here, the free evolution Hamiltonians $H_{i}^{0}=-\frac{\hbar^{2} \nabla^{2}}{2 m}+V_{i}(\vec{r})(i=1,2)$, the mean-field interaction Hamiltonians $H_{i}^{M F}=\frac{4 \pi \hbar^{2}}{m}\left(a_{i i}\left|\Psi_{i}(\vec{r}, t)\right|^{2}+a_{i j}\left|\Psi_{j}(\vec{r}, t)\right|^{2}\right)(i, j=1,2, i \neq j)$ and $a_{i j}$ is the scattering length between states $i$ and $j$ which satisfies $a_{i j}=a_{j i}$. Under the condition of weak coupling, i.e., $\Omega / \sqrt{\omega_{x}^{2}+\omega_{y}^{2}+\omega_{z}^{2}} \ll 1$ (in which $\Omega$ is the Rabi frequency, $\omega_{x}, \omega_{y}$ and $\omega_{z}$ are the trapping frequencies), the macroscopic wavefunctions can be written in form of the variational ansatz $\Psi_{i}(\vec{r}, t)=\psi_{i}(t) \Phi_{i}(\vec{r})(i=1,2)$ with amplitudes $\psi_{i}(t)=\sqrt{N_{i}(t)} e^{i \alpha_{i}(t)}$ 
and spatial distributions $\Phi_{i}(\vec{r})$. In this ansatz, the complex function $\psi_{i}(t)$ contains all timedependence in the macroscopic wave-function $\Psi_{i}(\vec{r}, t)$ and does not depend on the spatial coordinates. The symbols $N_{i}(t)$ and $\alpha_{i}(t)$ are the atomic population and phase of the $i$-th component, respectively. Due to the coupling is very weak, the spatial distributions vary slowly in time and are very close to the adiabatic solutions to the time-independent uncoupled case for GP equations (1), being slaved by the populations [11]. Thus, the amplitudes obey the nonlinear two-mode dynamical equations

$$
\begin{aligned}
& i \hbar \frac{d}{d t} \psi_{2}(t)=\left(E_{2}^{0}-\frac{\delta}{2}+U_{22}\left|\psi_{2}(t)\right|^{2}+U_{21}\left|\psi_{1}(t)\right|^{2}\right) \psi_{2}(t)+\frac{K}{2} \psi_{1}(t), \\
& i \hbar \frac{d}{d t} \psi_{1}(t)=\left(E_{1}^{0}+\frac{\delta}{2}+U_{11}\left|\psi_{1}(t)\right|^{2}+U_{12}\left|\psi_{2}(t)\right|^{2}\right) \psi_{1}(t)+\frac{K}{2} \psi_{2}(t) .
\end{aligned}
$$

The parameters $E_{i}^{0}=\int \Phi_{i}(\vec{r}) H_{i}^{0} \Phi_{i}(\vec{r}) d \vec{r}, U_{i j}=\frac{4 \pi \hbar^{2} a_{i j}}{m} \int\left|\Phi_{i}(\vec{r})\right|^{2}\left|\Phi_{j}(\vec{r})\right|^{2} d \vec{r}=U_{j i}$ and $K=\Omega \int \Phi_{1}(\vec{r}) \Phi_{2}(\vec{r}) d \vec{r}(i, j=1,2)$. The terms in $K$ describe the internal tunnelling between two BEC states. Whereas the terms in $U_{i j}$, which depend on the numbers of atoms in each BEC state, describe the mean-field interaction between atoms. When $U_{21}$ and $\delta$ equals zero, these coupled equations can also describe the BECs in a double-well potential and a nonlinear dimer [17]. Introducing a Bloch spin vector with following components

$$
u=\frac{\psi_{2}^{*} \psi_{1}+\psi_{2} \psi_{1}^{*}}{\psi_{1}^{*} \psi_{1}+\psi_{2}^{*} \psi_{2}}, v=-i \frac{\psi_{2} \psi_{1}^{*}-\psi_{2}^{*} \psi_{1}}{\psi_{1}^{*} \psi_{1}+\psi_{2}^{*} \psi_{2}}, w=\frac{\psi_{2}^{*} \psi_{2}-\psi_{1}^{*} \psi_{1}}{\psi_{1}^{*} \psi_{1}+\psi_{2}^{*} \psi_{2}} .
$$

Obviously, $u^{2}+v^{2}+w^{2}=1$. When the total atomic numbers $N_{T}=N_{1}+N_{2}=\psi_{1}^{*} \psi_{1}+\psi_{2}^{*} \psi_{2}$ is conserved, setting the Planck constant $\hbar=1$, the Bloch spin components satisfy

$$
\frac{d}{d t}\left(\begin{array}{l}
u \\
v \\
w
\end{array}\right)=\left(\begin{array}{ccc}
0 & \gamma+G w & 0 \\
-(\gamma+G w) & 0 & K \\
0 & -K & 0
\end{array}\right)\left(\begin{array}{l}
u \\
v \\
w
\end{array}\right)
$$

In above Bloch equation, the parameters satisfy $\gamma=E_{2}^{0}-E_{1}^{0}+N_{T}\left(U_{22}-U_{11}\right) / 2-\delta$ and $G=N_{T}\left(U_{22}+U_{11}-2 U_{12}\right) / 2$. Comparing the above equation with the one for the linear case $\left(U_{i j}=0\right)$ of equation (2), one can find that the mean-field interaction induces a shift $G w$ in the transition frequency and this shift is apparently proportional to the relative population $w$. 
Taking $\mid 1>$ as spin-up state and $\mid 2>$ as spin-down state, the above two-component BECs system can be regarded as an ensemble of quantum spin-1/2 particles. Thus, the longitudinal component $w$ of the pseudospin describes the relative population, and the transverse components $u$ and $v$ characterize the coherence. In this language, the effective Rabi frequency causes an effective transverse magnetic field $K$ along axis- $u$, the effective detuning induces an effective longitudinal magnetic field $\gamma$, and the mean-field interaction brings an effective longitudinal magnetic field $G w$ which depends on the longitudinal spin component. If one introduce a spin $\vec{S}=(u, v, w)$ to describe the coupled two-component BECs, the corresponding motion equation depicts the evolution can be written as

$$
\frac{d \vec{S}}{d t}=-\vec{S} \times \vec{B}_{e f f},
$$

where, the effective magnetic field $\vec{B}_{e f f}=(-K, 0,-\gamma-G w)$.

From definition of the Bloch spin components, we know that the above system can be described with only two independent variables. If we use the longitudinal spin component $w$ and the relative phase $\phi=\alpha_{2}-\alpha_{1}$ as independent variables, rescaling the time $K t$ to $t$, the motion equations

$$
\begin{aligned}
& d w / d t=-\sqrt{1-w^{2}} \sin \phi \\
& d \phi / d t=-\gamma / K-(G / K) w+w \cos \phi / \sqrt{1-w^{2}} .
\end{aligned}
$$

are equivalent to the Bloch's equation. The above equations are consistent with those derived from the secondary quantized model [10]. The form of the motion equation (6) is similar to the one for the condensates in a double-well potential coupled with tunnelling [17]. However, due to the difference between the original model, the physical means of the parameters are very different. For the condensates in a double-well potential, two condensates are well spatially separated, thus the mean-field interaction between two condensates can be ignored; but for the case of coupled two hyperfine-level condensates, the inter-condensate mean-field interaction play a very important role due to their significant overlap. One distinct result induced by this difference is the sign of the parameter $G$, for the case of condensates in a double-well potential, the sign is just determined by the sign of the scattering length; but 
for the other case, the sign is determined by the balance between the internal condensate and inter-condensate mean-field interactions.

\section{A. Phase-dependent Spontaneous Spin Polarization}

Before analyze the spontaneous spin polarization in coupled two-component BECs, we give a brief review of the spontaneous spin polarization in thermal gases and the spontaneous magnetization which is very similar to the spontaneous spin polarization $[1,3]$.

Usually, for a laser pumped thermal gas, if the spin exchange takes place faster than the spin relaxation and the coupling laser only excite some certain hyperfine levels of the ground state, spontaneous spin polarization occurs when the laser intensity is large enough. This novel bistable phenomenon involves three basic processes: laser pumping, spin exchange and spin relaxation. The imbalance of transition probabilities among different hyperfine states induced by the pumping laser will amplify the spin polarization. The spin exchange will keep the transition imbalance and does not destroy the spin polarization. However, the spin relaxation will decrease the spin polarization. In the case of the rates of spin exchange and laser pumping are larger than some certain threshold values, the thermal gas exists two metastable non-completely polarized states. This means the balance between spin relaxation and cooperation of laser pumping and spin exchange.

From the viewpoints of bistability, spontaneous spin polarization is similar to spontaneous magnetization. For a ferromagnetic system, if its temperature is below the Curie temperature, slowly changing the magnetic field from negative to positive, a first order phase transition occurs when the magnetic field sweeps through the zero point. At the zero field point, two metastable states with different spontaneous magnetization directions appear. The appearing state depends on the initial magnetization. Increasing the temperature above the Curie temperature, the spontaneous magnetization disappears, this corresponds to occurrence of a second order phase transition.

Under some certain conditions, both spontaneous spin polarization in a thermal gas and 
spontaneous magnetization in a ferromagnetic system appear novel bistability, and they just only depend on the related parameters. In the following, we find a novel character of the spontaneous spin polarization in laser coupled two-component BECs: phase dependence. That is, the spontaneous spin polarization in coupled two-component BECs depends on not only the related parameters but also the relative phase between two condensates.

Now, let us analyze the spontaneous spin polarization in coupled two-component BECs. It is well known, for a bounded dynamical system, the stable behavior usually oscillate around some stationary states. This indicates, the nonzero time-averaged value of a physical variable in a stable evolution requires that the system possesses at least a stationary state with nonzero value for this variable. Thus if there coexist multiple stationary states with nonzero spin polarization $w$ appearing in the coupled two-component BECs, the spontaneous spin polarization will appear. This means, one can explore the behavior of spontaneous spin polarization by analyzing the corresponding stationary states. The stationary states can be obtained from the stable fixed points of the system, which correspond to those solutions satisfying $d w / d t=0$ and $d \phi / d t=0$. In the region $[0,2 \pi)$ of the relative phase, we find two different modes of stationary states existing in the system: one is the equal-phase mode with zero relative phase $(\phi=0)$, the other one is the anti-phase mode with $\pi$ relative phase $(\phi=\pi)$. Small oscillations around those stationary states with nonzero spin polarization are special kinds of macroscopic quantum self-trapping (MQST) states, which have also been found in the condensates trapped in a double-well potential [17]. For the MQST sates, they only require the states oscillate around $d w / d t=0$, thus running-phase MQST states can appear. However, for the small oscillations around stationary states, their centers are stationary states which satisfy both $d w / d t=0$ and $d \phi / d t=0$, thus running-phase MQST states never appear. From the points of stability, running-phase MQST states are unstable, but all small oscillations around stationary states are all stable.

The number of fixed points and stationary states depend on the ratios $\gamma / K, G / K$ and the relative phase. For the equal-phase mode, only a fixed point exists when $G / K \leq 1$ and this fixed point is stable. When $G / K>1$, there are two stable fixed points and an unstable one 
for $(G / K)^{2 / 3}-(\gamma / K)^{2 / 3}>1$ and only one stable fixed point for $(G / K)^{2 / 3}-(\gamma / K)^{2 / 3}<1$. Saddle-node bifurcations occur at the points satisfying $(G / K)^{2 / 3}-(\gamma / K)^{2 / 3}=1$. In the left column of Fig. 1, we show the values for the longitudinal component of the fixed points in the equal-phase mode with different ratios $\gamma / K$ and $G / K$. For the anti-phase mode, the parametric dependence of fixed points and stationary states is very different. When $G / K \geq-1$, only a fixed point appears and it is stable. When $G / K<-1$, two stable fixed points and an unstable one exist for $(G / K)^{2 / 3}-(\gamma / K)^{2 / 3}>1$ and only one stable fixed point emerges for $(G / K)^{2 / 3}-(\gamma / K)^{2 / 3}<1$. Saddle-node bifurcations also occur at the points satisfying $(G / K)^{2 / 3}-(\gamma / K)^{2 / 3}=1$. The fixed points of the anti-phase mode with different ratios $\gamma / K$ and $G / K$ are exhibited in the right column of Fig. 1.

In the Fig. 1, the fixed points between a pair of bifurcation points with same ratio $G / K$ are unstable and the values for $d(\gamma / K) / d w$ at the bifurcation points equal zero. From the previous analysis, we find bistability exists in either the equal-phase mode or the anti-phase mode when the parameters obey $(G / K)^{2 / 3}-(\gamma / K)^{2 / 3}>1$. The appearance of bistability indicates the existence of spontaneous spin polarization in this coupled two-component BECs system. When $|K / G|<1$, and $\gamma / K$ goes through the bifurcation points which satisfy $(G / K)^{2 / 3}-(\gamma / K)^{2 / 3}=1$, the spin polarization of either the equal-phase mode or the anti-phase mode is discontinuous at the bifurcation points. This means a first-order phase transition of the spin polarization. It resembles that the first-order phase transition, spontaneous magnetization, in a ferromagnetic system below the Curie temperature takes place when vary direction of the magnetic field. The difference is that, spontaneous magnetization only occurs at the zero field point, however, spontaneous spin polarization occurs in the region between a pair of bifurcation points. Increasing $|K / G|$ to 1 , the spontaneous spin polarization vanishes, which corresponds to a second-order phase transition of the spin polarization. This is similar to spontaneous magnetization disappears in a ferromagnetic system when the temperature is increased to the Curie temperature. Thus, the ratio $K / G$ corresponds to the temperature in a ferromagnetic system and $|K / G|=1$ takes the role of 


\section{Curie temperature.}

Similar to the case of thermal atoms, spontaneous spin polarization can be induced by adjusting the coupling lasers. Additionally, due to the collisions among ultracold atoms can be controlled easily, spontaneous spin polarization in Bose condensed atoms can also be induced by adjusting the collision strength through bifurcation. This seems to adjust the temperature of a ferromagnetic system. Tuning the coupling laser with fixed intensity to a certain detuning satisfying $\gamma=0$, the bifurcation and the spontaneous spin polarization caused by the ultracold collisions can be obtained. For the equal-phase mode, only one stable

fixed point $w=0$ exists if $G / K<1$ and two new stable fixed points $w_{ \pm}= \pm \sqrt{1-(G / K)^{-2}}$ appear with the original one $w=0$ becomes unstable if $G / K>1$. This means a Hopf bifurcation takes place when $G / K=1$. The system goes from the Rabi regime $(G / K<1)$ into the spontaneous spin polarization regime $(G / K>1)$ through this Hopf bifurcation. However, for the anti-phase mode, the Hopf bifurcation occurs at $G / K=-1$. There is only one stable fixed point $w=0$ for $G / K>-1$ and two table fixed points $w_{ \pm}= \pm \sqrt{1-(G / K)^{-2}}$ with an unstable one $w=0$ for $G / K<-1$. Correspondingly, the parametric regime sustaining spontaneous spin polarization satisfies $G / K<-1$. The Hopf bifurcations in both equal-phase mode and anti-phase mode are shown in Fig. 2. The solid lines are stable equilibria (stationary states), the dot lines are unstable equilibria.

\section{B. Phase-dependent Bifurcation Delay}

The bifurcations obtained by analyzing the equilibria with fixed parameters are static bifurcations. For a real physical system, some parameters can be accurately tuned by turning knobs of experimental apparatus. When the parameters are swept through a static bifurcation point, an interesting phenomenon emerges: the system starting close to the initially stable equilibrium does not immediately react to the bifurcation. Furthermore, it remains for some time close to the unstable equilibrium, then fast falls into one of the newly formed stable equilibria. This has been named as bifurcation delay which has been found in a va- 
riety of physical systems [18]. The bifurcation delay, which might lead to hysteresis, is the response to the bistability.

Slowly varying some parameters, the coupled two-component BEC system also exhibits the phenomenon of bifurcation delay. For the equal-phase mode, fixed the effective detuning $\gamma=0$, slowly sweeping up the ratio $G / K$ from $R_{0}$ with sweeping rate $r$ (i.e., $G / K=R_{0}+r t$, $1 \gg r>0$ ), choosing $R_{0}<1$ and the initial state close to the stable equilibrium, the system evolves along the unstable equilibrium for a period of time after the ratio sweeping through the static bifurcation point $(G / K=1)$, then it quickly goes into a small oscillations around one of two new stable equilibria. The equilibrium, which the system evolves around lastly, determines by the state at the static bifurcation point. The system evolves around the up branch lastly when this state is close to the up branch; otherwise, the system evolves around the down branch. When $R_{0}>1$, slowly sweeping down the ratio through the static bifurcation point with initial state close to one of two stable equilibria, the system evolves near the stable equilibrium before it sweeps through the static bifurcation point, then it goes into a small Rabi oscillation around the ordinary equilibrium $(w=0)$. For the same sweeping rate, averaging the small oscillations, the process of sweeping up and down generates a loop in the plane extended by $G / K$ and $w$. The area enclosed in the loop increases with the sweeping rate. This means that the energy exchanged between the atoms and the environments increases with the sweeping rate. The bifurcation delay in the equal-phase mode with different sweeping rate is shown in Fig. 3. For the anti-phase mode, a similar behavior can be observed near the static bifurcation point $G / K=-1$.

\section{Experimental Possibilities}

Based upon the works of JILA [5] and LENS [6], we now discuss experimental possibilities of observing the spontaneous spin polarization and bifurcation delay predicted in above. Using the developed experimental technique [5,6], one can prepare two BECs in the $\mid F=1, m_{F}=-1>$ and $\mid 2,1>$ hyperfine spin states of ${ }^{87} R_{b}$ which are coupled by introduc- 
ing a two-photon pulse with the two-photon Rabi-frequency $\Omega$ and a finite detuning $\delta$. In the case of pure condensates which has been analyzed in previous, controlling the parameters $K$ and $\gamma$ can be realized by adjusting Rabi frequency and detuning of the coupling lasers, respectively. Tuning the parameter $G$ can be accomplished by varying the scattering lengths with Feshbach resonances [9]. The time-evolution of longitudinal and transverse spin components can be measured with the state-selective absorption imagining and the Ramsey interference, respectively [16].

Thus, to observe spontaneous spin polarization, one just need choose proper fixed values for Rabi-frequency $\Omega$, detuning $\delta$ and scattering lengths satisfying $(G / K)^{2 / 3}-(\gamma / K)^{2 / 3}>1$. To observe bifurcation delay, one has to fix Rabi-frequency $\Omega$ and detuning $\delta$ and slowly vary scattering lengths (or fix scattering lengths and detuning and slowly tune Rabi-frequency) through a static bifurcation point. There are two ways to observe these behaviors, one way is by directly observing the stationary states, the other way is by observing small oscillations around stationary states. Observing stationary state behavior may be not easy, because the relaxation time of a pure condensate is much longer than that of a thermal gas and time scale of this relaxation process, which is relevant to real experimental systems including finite temperature thermal clouds, is still an open problem. Fortunately, due to the averaged center of small oscillations is very close to the surrounded stationary state, the averaged center becomes a good understudy.

\section{SUMMARY AND DISCUSSION}

Summary, due to the coherent ultracold collision among condensed bosonic atoms, the bifurcation and the spontaneous spin polarization in coupled two-component BECs rely on both relative phase and physical parameters. These phenomena are different from those only determined by physical parameters, we name them as phase-dependent bifurcation and phase-dependent spontaneous spin polarization, respectively. For zero effective detuning $\gamma$, Hopf bifurcation and bifurcation delay can be induced by a Feshbach resonance in either 
the equal-phase mode or the anti-phase mode. The system falls into the spontaneous spin polarization regime from the Rabi regime after a bifurcation occurs. The appearance of bifurcation delay indicates the existence of metastability and hysteresis. Because of the inherently quantum coherence and superposition of two condensates, this kind of quantum metastability and hysteresis might open the door to storage quantum data with Bose condensed atoms [19].

In this article, we have focused on the novel phenomena in the case of constant parameters. Now, we give a brief discussion about the effects of the implicit time-dependence of the parameters. In the weak coupling case, the spatial functions $\Phi_{i}(\vec{r})$ weakly depend on the population difference. Thus these functions weakly rely on time implicitly when the population difference varies with time. The numerical results in Ref. [11] show the overlap between two condensates keeps nearly unchanged and the chemical potential difference is approximately a linear function of the population difference when populations are varied. This indicates that the parameters slowly fluctuate around some certain constants with very small amplitudes when the population oscillates. Thus the real population oscillation slightly depart from the one with constant parameters.

We also note that bistability and anti-phase mode are relative to population self-trapping states and $\pi$-states in a double-well Bose condensates or a nonlinear dimer [17]. The appearance of bistability means the existence of metastable self-trapping states. But not all self-trapping states exhibit bistability, such as running-phase self-trapping states. Additionally, there exist distinct difference in physical models, analysis methods and discussed phenomena. For the physical models, two condensates in a double-well potential are well spatially separated, thus mean-field interaction between two condensates is negligible. However, mean-field interaction between two hyperfine-state condensates acts an important role due to their significant overlap. For the analysis methods, the authors of Ref. [17] solve motion equations for some certain initial conditions with numerical approach or analyze stationary states of symmetric case. In this article, from the viewpoints of bifurcation, we exactly analyze not only the fixed points themselves but also their stability for arbitrary 
parameters. For the discussed phenomena, the works in Ref. [17] are applied to population self-trapping and macroscopic quantum tunnelling, our work firstly explores the spontaneous spin polarization and bifurcation delay in laser pumped Bose condensed dilute atomic gases.

\section{Acknowledgment}

The work is supported by foundations of MPI-PKS and Chinese NSFC (Grant No. 10275023 and 10274093), National Fundamental Research Program (Grant No. 2001CB309300). We thanks Dr. J. Feng of WIPM for useful comments.

\section{Figure caption}

Fig. 1 The fixed points for the system with different ratios $\gamma / K$ and $G / K$. The numbers labelled on the lines are values for $G / K$.

Fig. 2 The static Hopf bifurcation and the spontaneous spin polarization.

Fig. 3 The bifurcation delay in the equal-phase mode for different values of sweeping rate which are labelled on the lines. 


\section{REFERENCES}

[1] N. Fortson and B. Heckel, Phys. Rev. Lett. 59, 1281 (1987); W. M. Klipstein, S. K. Lamoreaux, and E. N. Fortson, Phys. Rev. Lett. 76, 2266 (1996); A. Andalkar, et. al., Phys. Rev. A 65, 023407 (2002).

[2] H. M. Gibbs, et al., Phys. Rev. Lett. 36, 113 (1976); T. Yabuzaki, et al., Phys. Rev. A 29, 1964 (1984).

[3] M. Rao, H. R. Krishnamurthy, and R. Pandit, Phys. Rev. B 42, 856 (1990).

[4] J. Stenger, S. Inouye, D. M. Stamper-Kurn, H. -J. Miesner, A. P. Chikkatur, and W. Ketterle, Nature 396, 345 (1998); C. J. Myatt, E. A. Burt, R. W. Ghrist, E. A. Cornell, and C. E. Wieman, Phys. Rev. Lett. 78, 586 (1997).

[5] D. S. Hall, M. R. Matthews, J. R. Ensher, C. E. Wieman, and E. A. Cornell, Phys. Rev. Lett. 81, 1539 (1998); D. S. Hall, M. R. Matthews, C. E. Wieman, and E. A. Cornell, Phys. Rev. Lett. 81, 1543 (1998).

[6] A. Smerzi, et. al., Eur. Phys. J. B 31, 457 (2003).

[7] K. Burnett, P. S. Julienne, P. D. Lett, E. Tiesinga, and J. Williams, Nature 416, 225 (2002); J. Weiner, V. S. Bagnato, S. Zilio, and P. S. Julienne, Rev. Mod. Phys. 71, 1 (1999).

[8] J. R. Anglin and W. Ketterle, Nature 416, 211 (2002); F. Dalfovo, S. Giorgini, L. P. Pitaevskii, and S. Stringari, Rev. Mod. Phys. 71, 463 (1999).

[9] S. Inouye, et. al., Nature 392, 151 (1998); Ph. Courteille, R. S. Freeland, and D. J. Heinzen, Phys. Rev. Lett. 81, 69 (1998); S. L. Cornish, et. al, Phys. Rev. Lett. 85, 1795 (2000); S. Chu, Nature 416, 206 (2002).

[10] S. Kohler and F. Sols, Phys. Rev. Lett. 89, 060403 (2002).

[11] J. Williams, et al., Phys. Rev. A 59, R31 (1999); J. Williams, Ph.D thesis, JILA and 
University of Colorado (1999).

[12] C. Lee, W. Hai, L. Shi, X. Zhu, and K. Gao, Phys. Rev. A 64, 053604 (2001); W. Hai, C. Lee, G. Chong, and L. Shi, Phys. Rev. E 66, 026202 (2002); C. Lee, W. Hai, X. Luo, L. Shi, and K. Gao, arXiv: cond-mat/0206134 (2002).

[13] L. -M. Kuang and Z. -W. Ouyang, Phys. Rev. A 61, 023604 (2000); B. Hu and L. -M. Kuang, Phys. Rev. A 62, 023610 (2000); L. -M. Kuang, Z. -Y. Tong, Z. -W. Ouyang, and H. -S. Zeng, Phys. Rev. A 61, 013608 (2000); L. -M. Kuang, J. -H. Li and B. Hu, J. Opt. B: Quantum Semiclass. Opt. 4, 295 (2002).

[14] R. J. Ballagh, K. Burnett, and T. F. Scott, Phys. Rev. Lett. 78, 1607 (1997); M. Ö. Oktel and L. S. Levitov, Phys. Rev. Lett. 83, 6 (1999); Y. Wu and X. Yang, Phys. Rev. A 62,013603 (2000).

[15] K. Marzlin, W. Zhang, and E. M. Wright, Phys. Rev. Lett. 79, 4728 (1997); R. Dum, J. I. Cirac, M. Lewenstein, and P. Zoller, Phys. Rev. Lett. 80, 2972 (1998).

[16] H. J. Lewandowski, et al., Phys. Rev. Lett. 88, 070403 (2002); M. Ö. Oktel and L. S. Levitov, Phys. Rev. Lett. 88, 230403 (2002); J. N. Fuchs, D. M. Gangardt, and F. Laloë, Phys. Rev. Lett. 88, 230404 (2002); J. E. Williams, T. Nikuni, and C. W. Clark, Phys. Rev. Lett. 88, 230405 (2002); J. M. McGuirk, et al., Phys. Rev. Lett. 89, 090402 (2002); T. Nikuni, J. E. Williams, and C. W. Clark, Phys. Rev. A 66, 043411 (2002).

[17] M. R. Andrews, et al., Science 275, 637 (1997); C. Orzel, et al., Science 291, 2386 (2001); A. Smerzi, et al., Phys. Rev. Lett. 79, 4950 (1997); S. Raghavan, et al., Phys. Rev. A 59, 620 (1999); S. Raghavan, A. Smerzi, and V. M. Kenkre, Phys. Rev. A 60, R1787 (1999); I. Marino, et al., Phys. Rev. A 60, 487 (1999); A. Smerzi and S. Raghavan, Phys. Rev. A 61, 063601 (2000); V. M. Kenkre and D. K. Campbell, Phys. Rev. B 34, 4959 (1986); G.P.Tsironis and V.M. Kenkre, Phys. Lett. A 127, 209 (1988).

[18] P. Mandel and T. Erneux, Phys. Rev. Lett. 53, 1818 (1984); N. Berglund and H. Kunz, 
Phys. Rev. Lett. 78, 1691 (1997); N. Berglund and H. Kunz, J. Phys. A 32, 15 (1999).

[19] E. M. Chudnovsky, Science 274, 938 (1996); P. C. E. Stamp, Nature 383, 125 (1996). 